\title{
Centre et périphérie \\ La double logique de l'occupation des territoires au Québec
}

\author{
Marc-Urbain Proulx
}

\begin{abstract}
Au-delà des débats ponctuels sur l'exode rural, la forêt boréale, les gisements éoliens, les bassins hydroélectriques, l'uranium

La périphérie nordique québécoise a toujours évoqué un immense bassin de ressources naturelles que seuls les plus intrépides des individus et des entreprises se risquent à explorer, à extraire et à valoriser
\end{abstract} et autres minéraux, la

risquent à explorer, à extraire et à valoriser. L'épopée de Radisson et son compagnon Desgroseillers s'avère mythique à cet égard. Ces aventuriers ont route maritime de l'océan Arctique, se positionnent quelques préoccupations sociétales fondamentales à propos des territoires de la périphérie nordique du Québec. Il existe bien sûr l'enjeu central concerné par les droits de propriété des nations autochtones. Il y a aussi l'important défi collectif relié à la protection de l'environnement et au renouvellement des ressources naturelles dans un esprit de durabilité. La demande du marché mondial à l'égard des ressources naturelles représente aussi une dimension incontournable de la problématique nordique.

En outre, les modalités spatio-économiques (géo-économiques) en regard de l'occupation des divers territoires qui composent le Nord du Québec représentent un enjeu d'importance stratégique. Nous le traiterons dans ce texte par l'établissement des jalons de base d'un cadre de référence pour l'ensemble du Québec, en revisitant les deux logiques spatioéconomiques classiques, soit la centralité et l'accessibilité.

Mis à part Voltaire et ses «quelques arpents de neige », la périphérie nordique québécoise a toujours évoqué un immense bassin de ressources naturelles que seuls les plus intrépides des individus et des entreprises se atteint la Baie James, une première fois en utilisant les rivières Outaouais et Harricana, et quelques années plus tard directement par la mer à travers vents et marées via le détroit d'Hudson. L'expédition du Père Albanel qui a exploré le lac Mistassini, avant de descendre la rivière Rupert jusqu'à la Baie James, n'est pas banale non plus. Elle a permis de marquer l'itinéraire de la fabuleuse «route des fourrures » qui empruntait la rivière Chicoutimi, les lacs Kénogami et Pékouagami, avant de sillonner la rivière Ashuamushan vers les territoires nordiques. Bref, l'histoire est remplie de magnifiques exemples de prouesses qui furent nécessaires pour accéder au Nord québécois. En réalité, l'accessibilité aux lieux et aux milieux s'avère une composante problématique de base à toute volonté d'analyse des conditions de l'occupation des territoires de la périphérie nordique du Québec.

Pour bien saisir cette logique spatioéconomique reliée à l'accessibilité au Nord du Québec, notre analyse mettra d'abord en relief la logique classique de la centralité à travers ses mouvances spatiales très actuelles. Nous verrons que la polarisation des activités économiques forme progressivement une vaste concentration déconcentrée dans le sud-est, zone qui s'inscrit comme le véritable centre du 
Québec. Par la suite, notre analyse s'appliquera à illustrer les formes épousées par l'occupation actuelle et anticipée des territoires de la périphérie nordique.

\section{De comptoir à métropole}

La Ville de Québec fut historiquement un véritable avant-poste de conquête territoriale en Amérique du Nord, pour le bénéfice de la métropole française. $\mathrm{Ce}$ «comptoir continental» a permis à l'époque de drainer les ressources rapidement exploitables d'une très vaste périphérie, en remontant les cours d'eau, en plantant des drapeaux et des croix, en traitant avec les autochtones, en établissant d'autres avant-postes à Montréal, à LongSault, à Sault-Sainte-Marie, à Chicoutimi.

Après ce premier écrémage de la périphérie dite Nouvelle - France, Québec s'est inscrit aussi comme un lieu de gouverne de l'occupation plus intensive des territoires par la colonisation des terres. La ville est ainsi lentement devenue le principal marché de la croissante production agricole, alors que son industrie navale fut tournée vers l'extérieur au même titre que le lucratif commerce des fourrures. Avec l'exploitation systématique de la forêt pour le compte de l'Angleterre au début du XIXième siècle, la ville de Québec a atteint son apogée comme principal centre urbain.

\section{Quatre facteurs ont occasionné le déplacement vers Montréal des principales forces de la polarisation des activités et de la population}

\section{$\underline{\text { Renversements spatio-économiques }}$}

Quatre facteurs ont occasionné le déplacement vers Montréal des principales forces de la polarisation des activités et de la population. D'abord, l'élite d'affaires anglaise qui est arrivée après la conquête militaire de 1760 s'est largement établie à Montréal, en prenant le contrôle de la traite de fourrures et en lançant par la suite de nouvelles activités économiques notamment des fabriques et des industries. Cette diversification économique a considérable-ment dirigé les exportations québécoises vers les villes limitrophes des États-Unis devenues un important marché relativement facile à desservir à partir de Montréal. Troisièmement, l'urbanisation forte $\mathrm{du}$ Québec au XIXème siècle s'est particulièrement effectuée dans la large plaine du Saint-Laurent autour de Montréal offrant ainsi à ce centre urbain une mission importante dans la desserte de services supérieurs à son prospère hinterland. Finalement et non le moindre, la canalisation du fleuve SaintLaurent jusqu'à Montréal a permis à cette ville de devenir, à partir de 1850 , le principal point de transbordement des marchandises au Québec. La construction des canaux et ensuite des chemins fer a substantiellement soutenu ce renversement spatio-économique qui a déplacé à Montréal la principale place d'affaire du Québec. Bien positionnée, cette ville est alors devenue la métropole du Canada, obtenant même l'établissement du premier parlement canadien. Malgré la construction du canal Érié comme lien direct entre la côte Atlantique et l'intérieur du territoire du côté américain de la frontière, la croissance forte de Montréal s'est poursuivie jusque vers les années 1950, avant que Toronto prenne le relais de la forte polarisation à la faveur de la puissante industrialisation de la région des Grands Lacs et de la canalisation du fleuve Saint-Laurent jusqu'à ce vaste bassin maritime prospère.

À cet effet de double renversement spatial historique, d'abord entre Québec et Montréal et ensuite entre Montréal et Toronto, signalons qu'il existe un important débat non épuisé au 
Québec à propos des causes du déclin relatif de Montréal depuis un demi- siècle. Débat qui est repris actuellement par certains analystes autour de l'envergure réelle de la reprise économique très actuelle de la métropole du Québec, en comparaison avec les autres pôles nord-américains y compris Boston, Québec, Vancouver. Mais ce débat n'est pas notre propos. Si les performances des divers centres urbains qui polarisent les activités dans l'espace s'avèrent pertinentes à comparer sur le grand espace canadien ou ailleurs, le phénomène contemporain de ladite métropolisation nous convie dans ce texte à un autre type d'analyse. Nous verrons qu'au Québec, la grande région métropolitaine possède sa contrepartie périphérique dont la composante nordique. La dualité " centre périphérie 》 si chère à l'analyse spatioéconomique, épouse en réalité des formes bien spécifiques et distinctes. Dualité de formes qui est notamment très bien illustrée par les zones de rayonnement des centres urbains ${ }^{1}$.

\section{Carte 1}

Les duales aires de rayonnement des agglomérations urbaines

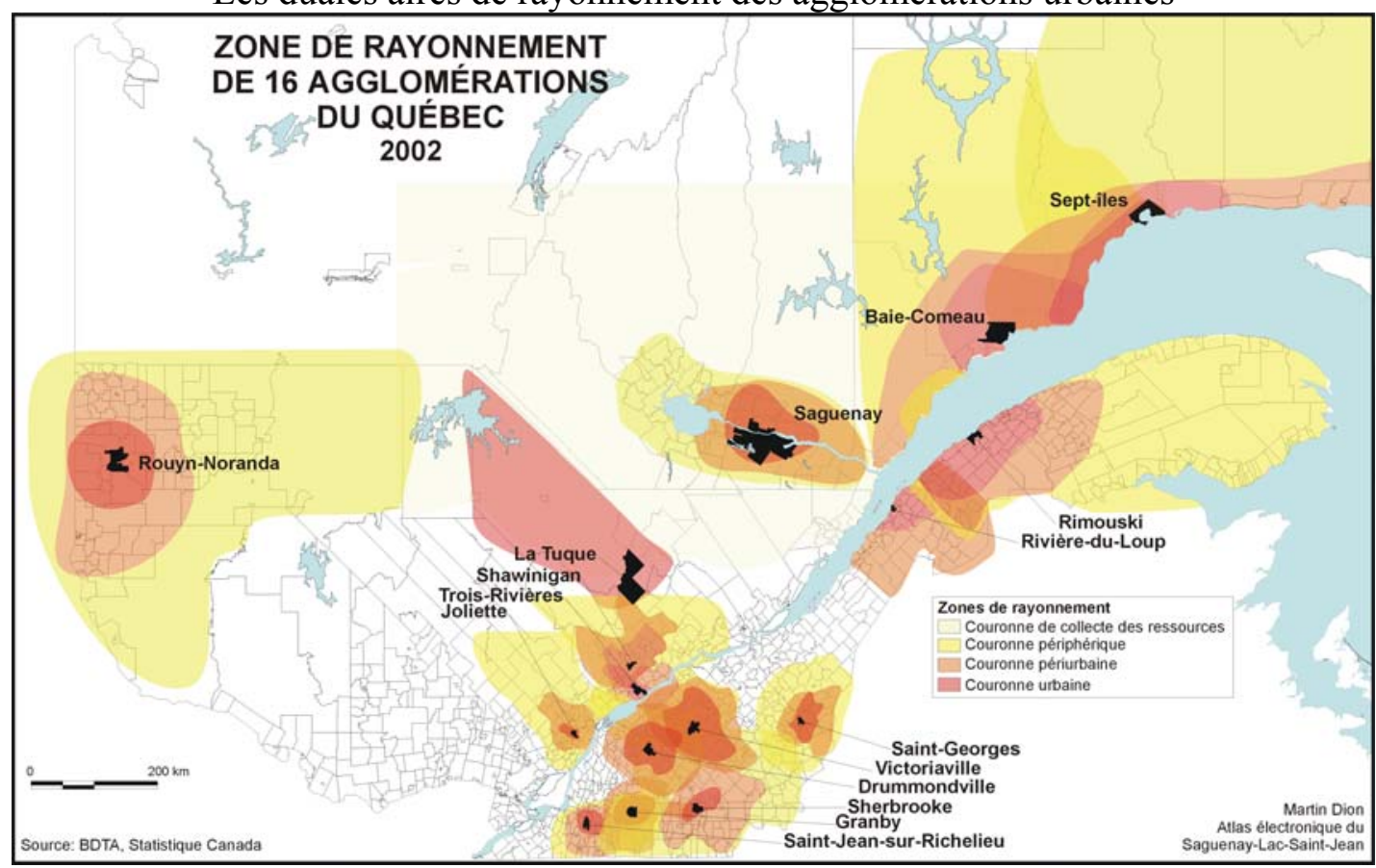

On constate en effet sur la carte no 1 que l'aire d'influence des centres urbains dessine deux types d'aires bien distinctes. Elles sont contigües dans le centre du Québec et plutôt relâches en périphérie.

\section{$\underline{\text { La métropolisation }}$}

On sait qu'à l'échelle mondiale, la population et les activités économiques sont de plus en plus concentrées au sein de quelques 300 vastes régions métropolitaines interreliées les unes aux autres. Si vastes que ces régions contiennent souvent plusieurs centres urbains en épousant des formes territoriales inédites souvent appelées mégapoles. En conséquence, saisir concrètement ce phénomène de métropolisation ouvre sur une analyse davantage orientée sur la structuration multipolaire concrète de régions urbaines dites Global City-Regions ${ }^{2}$.

Selon cette perspective, observer la mouvance actuelle des mégapoles européennes, 
américaines et asiatiques devient une activité fort intéressante. Car les formes territoriales spécifiques et diversifiées qui émergent alors à l'analyse métropolitaine font apparaître des jumeaux tels que Milan - Turin, des triplets tels que Rio Janeiro - Bello Horizonte - Sao Paulo, des corridors polycentriques tels que celui de la Californie, des arcs comme celui de la Méditerranée entre l'Espagne et l'Italie en passant par le sud de la France. Aussi, le croissant territorial composé de Tokyo - Séoul - Beijing - Shanghai - Hong Kong illustre parfaitement cette forme mégapolitaine complexe structurée par des métropoles mondiales entrecoupées de pôles et de zones à divers contenus, natures et dimensions. À cet effet de "mégapolisation » de la dynamique économique planétaire, la forme de l'archipel s'avère largement référée dans la littérature scientifique. Plusieurs analystes utilisent cette image pour illustrer des ensembles en Amérique, en Asie, en Afrique, en Europe.

L'espace canadien ne contient pas de mégapole. Cependant le phénomène métropolitain s'avère fort bien illustré par la grande région urbaine de Toronto qui contient non seulement des pôles périurbains et des satellites dans la frange urbaine mais aussi des centres intermédiaires bien connectés comme London, Windsor, Kitchener, Hamilton, Oshawa, etc ${ }^{3}$. Il s'agit certes d'un type d'archipel.

\section{Nous assistons à un processus} relativement complexe d'étalement urbain et de polarisation que certains désignent par le terme métapolisation

Dans le cas du Québec, le déploiement territorial des forces de la concentration et de la dispersion permet de saisir la métropolisation sous un angle particulier ${ }^{4}$. Nous assistons à un processus relativement complexe d'étalement urbain et de polarisation que certains désignent par le terme métapolisation ${ }^{5}$, c'est-à-dire une urbanisation diffuse qui concentre la population et les activités au sein d'une vaste zone composée de divers territoires réticulés qui lui donnent forme. Car le phénomène métropolitain qui bat son plein au Québec comme ailleurs touche plusieurs pôles québécois de différentes tailles dont l'imbrication d'ensemble apparaît désormais moins régie par la hiérarchie urbaine classique que par d'autres lois spatioéconomiques difficiles à modéliser puisque constamment évolutives. Nous pouvons néanmoins saisir les points d'appui et visionner l'armature globale. Voyons un peu.

\section{$\underline{\text { La métapole québécoise }}$}

Certes, les pôles principaux que sont Montréal, Québec et Gatineau (Ottawa) avec leurs centres périurbains et leurs satellites réciproques s'inscrivent chacun comme un phénomène métropolitain à part entière. Dans une moindre mesure, il en est de même pour les régions métropolitaines de Sherbrooke, de Trois-Rivières et aussi de Drummondville, Rivière-du-Loup, Saint-Hyacinthe, Shawinigan. À travers ces principaux centres urbains qui structurent l'armature de la grande région sud-est du Québec, la concentration diffuse ou déconcentrée des activités économiques s'ancre aussi sur de nombreux petits pôles comme Sorel, Bromont, Joliette, Thetford Mines, Sainte-Marie, Plessisville, Montmagny, etc. Parmi ceux-ci, certains pôles s'avèrent actuellement en véritable explosion tels que Saint-Georges, Granby, Victoriaville, Saint-Sauveur en illustrant des taux de croissance urbaine les plus élevés du Québec. Les forces qui positionnent les lieux dans cette zone métapolitaine résident principalement dans leur centralité à travers les établissements humains dispersés. 
Carte no 2

Le croissant manufacturier du Québec

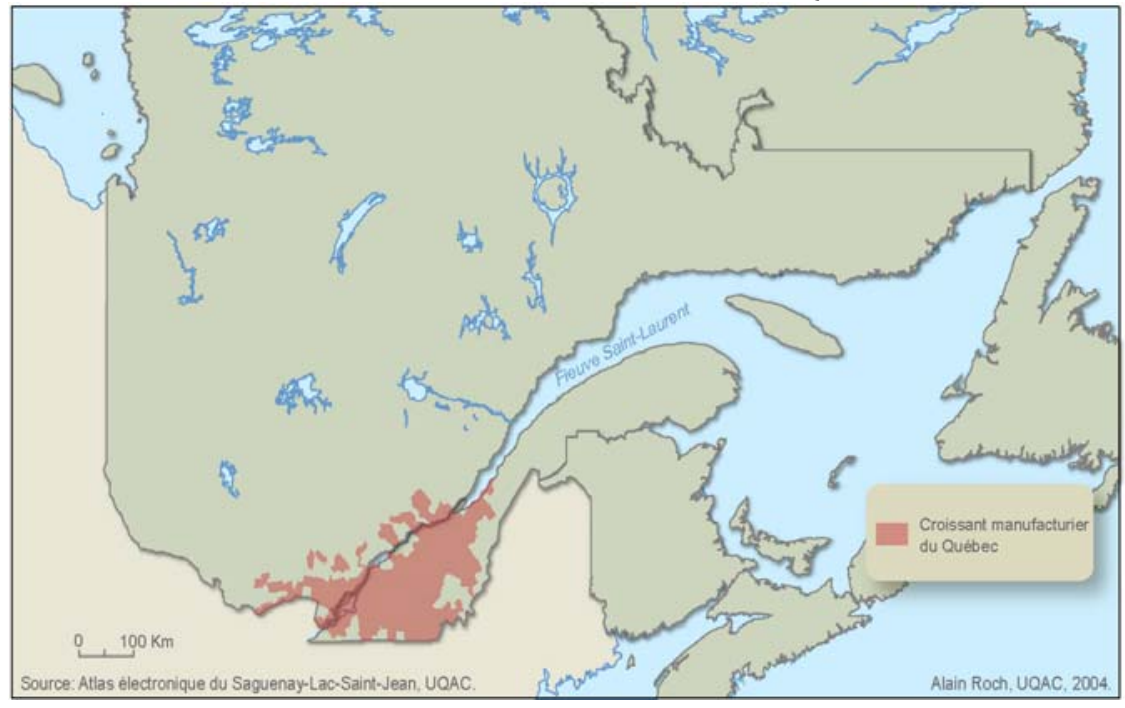

Ce vaste tissu métapolitain québécois qui devient en réalité le véritable «centre déconcentré » du Québec s'étale progressivement à densités variables en suivant, par sauts d'urbanisation, les corridors de l'Outaouais, des Laurentides, de la BasseMauricie, de la Beauce, du Bas-Saint-Laurent et aussi de Charlevoix dans une moindre mesure. Bien que la dispersion de tous ces pôles primaires, secondaires et tertiaires soit importante dans ce sud-est qui est de fait le centre-du-Québec, nous percevons clairement un " effet d'ensemble", ne serait-ce que le système de transport de plus en plus fluide et dense. Densité à saisir en matière de personnes mais aussi de marchandises. Aussi, la carte no 1 nous permet de constater que contrairement à la périphérie québécoise, cette métapole en

\section{La périphérie nordique}

En dehors de la vallée du Saint-Laurent, il existe seulement quatre zones agricoles dont les établissements ont pu permettre l'émergence de pôles urbains sur la base des marchés locaux centraux. Il s'agit de la couronne agricole autour du lac Saint-Jean, du progrès illustre des aires de rayonnement des pôles relativement rapprochés qui laissent peu de zones orphelines. Étant donnée la vocation manufacturière dominante de cette zone métapolitaine, nous avons à cet effet statistiquement circonscrit un «croissant manufacturier » qui donne susbtance ${ }^{6}$ à ce phénomène de centralité polycentrique dans le sud-est du Québec (carte no 2).

Cette métapole québécoise contient aussi plusieurs centres de villégiatures, beaucoup de villages ruraux à croissance urbaine, des technopoles comme celui de Saint-Hyacinthe ou de la Cité du multimédia, une capitale nationale et évidemment la grande région montréalaise de services supérieurs spécialisés.

plateau du Saguenay, de la vallée de la rivière Matapédia et aussi, dans une moindre mesure, $\mathrm{du}$ corridor côtier du Lac Témiscamingue. Dans ces zones jadis colonisées, les rentes agricole et forestière ont créé les surplus suffisants pour occasionner la concentration 
$d^{\prime}$ 'entreprises de services et de commerces dans des lieux centraux tels que Amqui, Causapscal, Ville Marie, Chicoutimi, Roberval, etc. Pour le reste, les villes et villages de la périphérie nordique du Québec sont largement tributaires de la présence de ressources naturelles, à la manière de la Gaspésie.

En réalité, les forces qui imposent l'établissement de lieux et de milieux en périphérie québécoise résident principalement dans l'accès aux bassins et gisements de ressources naturelles qui représentent le véritable facteur explicatif de la grande dispersion spatiale des lieux et des milieux. Aussi, la desserte des collectivités autochtones en biens et services s'inscrit dans cet esprit d'extension de la structure de peuplement. Ce principe moteur de l'accessibilité dessine sur les territoires périphériques du Québec des formes territoriales distinctes, originales et particulières. Le chapelet de petits lieux gaspésiens en représente un bel exemple à partir du pôle Rimouski. Voyons un peu pour la périphérie nordique.

\section{$\underline{\text { Un arc nordique de pôles secondaires }}$}

Le phénomène spatio-économique majeur qui marque la structure de peuplement en périphérie nordique québécoise s'avère concerné par la présence d'avant-postes établis tels de véritables tremplins pour lancer l'occupation territoriale. En réalité, ce sont des pôles de croissance contenant des activités telles que des services spécialisés, des usines de transformation de ressources, des commerces de gros et de détails, des administrations publiques et privées, etc. à partir desquels s'effectuent l'exploitation des ressources naturelles ainsi que la diffusion du développement sur les territoires (carte 3).

Ces avant-postes s'inscrivent généralement comme de points de rupture spatiale aux embouchures de rivières, aux croisements de routes, aux terminaux des voies de transport. Ils deviennent des relais de pénétration nordique, grâce à la présence de différentes activités reliées certes au transbordement, à la traite et au transit de marchandise, mais aussi à la desserte de services de santé, d'éducation, de sports, de finances, de commerces, de génie, d'architecture, de comptabilité et autres spécialités motrices de développement. Les principaux spécimens de ces pôles économiques sont en réalité Sept-Îles, BaieComeau, Saguenay, Amos, Rouyn.

Dans leur périphérie nordique de rayonnement, ces pôles, secondaires par rapport à Montréal, Québec et Gatineau, forment ensemble un arc qui s'inscrit comme une forme territoriale originale (carte 3). Arc dont la spécificité spatio-économique d'ensemble concerne davantage la vocation nordique commune de ses composantes urbaines plutôt que leurs interrelations socio-économiques déjà existantes certes, mais encore peu développées. Relations et échanges horizontaux qui demeurent à intensifier entre les pôles de l'arc, dans un esprit de complémentarité et d'interdépendance face à l'enjeu de l'appropriation collective réciproque de leur destin nordique commun.

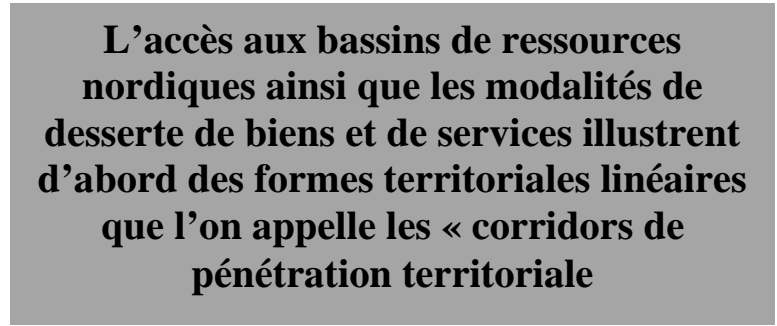

Des corridors de pôles tertiaires

Selon ce schéma spatio-économique, l'accès aux bassins de ressources nordiques ainsi que les modalités de desserte de biens et de services illustrent d'abord des formes territoriales linéaires que l'on appelle les « corridors de pénétration territoriale » (carte $3)$. 
Carte 3

Avant-postes et corridors de pénétration de la périphérie nordique

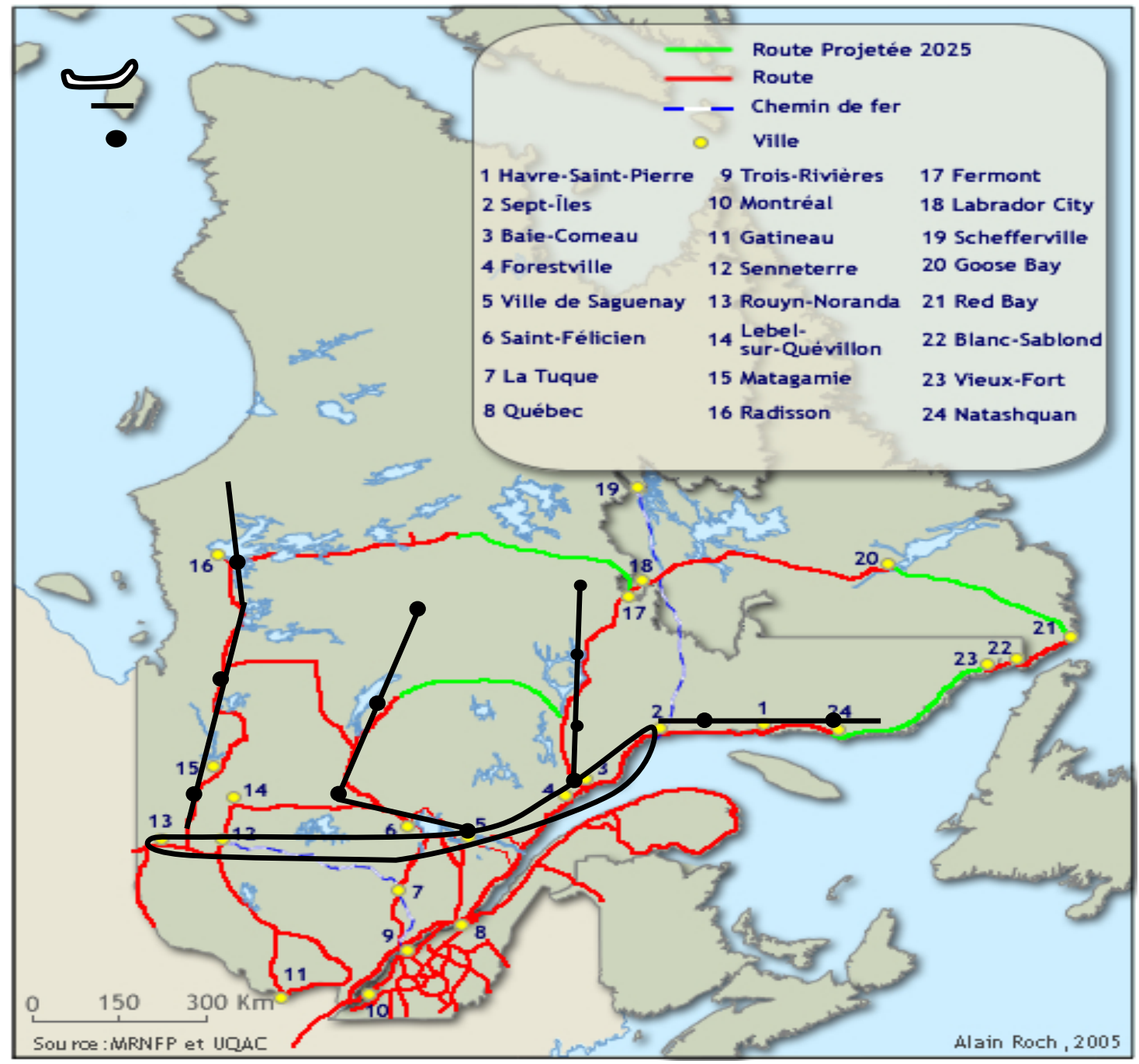

Ceux-ci épousent actuellement cinq axes, soit " Rouyn - Amos - Radisson » ; " Saguenay Saint-Félicien - Chibougamau - Némiscau »; «Baie-Comeau - Manic - Fermont - Goose Bay» ; «Sept-Îles - Havre-Saint-Pierre Natashquan» ainsi que «Sept-Îles Shefferville » établi que par une voie ferrée. Distincts dans leur forme réciproque, ces corridors partagent une nature commune et des contenus similaires.

C'est ainsi qu'à partir des avant-postes initiaux de l'arc nordique (Sept-Îles,
Saguenay, Amos...), les corridors de transport structurent l'espace par l'établissement de relais plus avancés qui deviennent des pôles tertiaires et quaternaires. Ces derniers sont localisés à des points précis déterminés généralement, mais pas toujours, par les modalités de transport qui favorisent la localisation de commerces, de services et aussi d'activités d'extraction des ressources naturelles. D'autres tronçons de ces corridors peuvent être anticipés, notamment sur l'axe côtier de la Baie d'Hudson afin d'interconnecter les villages Inuits entre eux 
ou pour relier le Lac Mistassini à Caniapiscau en passant par les Monts Ostih, ou encore pour joindre Natashquan et Blanc Sablon sur la Basse-Côte-Nord.

De ces corridors plus ou moins verticaux qui pointent vers le nord pour pénétrer la périphérie à partir de l'arc nordique, des couronnes plutôt horizontales ou transversales sont envisagées afin de permettre l'accessibilité à de vastes zones encore enclavées. Existe déjà la couronne «Chibougamau - Némiscau » qui conduit au territoire de la Baie-James. Aussi, la couronne " Goose Bay- Blanc Sablon " s'avère en construction actuellement au Labrador. Par ailleurs, deux autres couronnes sont anticipées dans le futur rapproché (carte 3). Il s'agit du lien routier qui sera éventuellement construit entre le Lac Mistassini et le réservoir Manicouagan ainsi que celui que l'on tissera un jour peut-être rapproché entre le réservoir de Caniapiscau et Labrador City. Toutes ces couronnes nordiques réelles et anticipées s'ajouteront à une couronne beaucoup plus ancienne en Gaspésie, soit Rimouski, Gaspé, Bonaventure, Matapédia.

\section{$\underline{\text { Un semis de petits lieux }}$}

À partir des pôles tertiaires et quaternaires que représentent Amos, Chibougamau, HavreSaint-Pierre, Val d'Or, Saint-Félicien, Blanc Sablon, pointent des voies de transport et de rayonnement vers l'intérieur de la périphérie truffée de petits lieux et petits milieux dispersés sur de vastes surfaces en fonction de la dotation territoriale en ressources. Notons que plusieurs de ces petits établissements humains ne sont pas reliés au système routier principal en étant desservis par des moyens de transport maritime et aérien, notamment plusieurs collectivités autochtones.

Ce semis de lieux et de milieux de petites ou très petites tailles devient le cinquième phénomène spatio-économique de la périphérie nordique du Québec. Ils incluent évidemment les collectivités autochtones telles que Chissasibi, Lac Simon, Umiujaq, La Tabatière. Des dizaines de petits centres ont ainsi émergé, souvent de manière explosive, souvent au milieu de nulle part mais généralement à proximité d'un bassin de ressources naturelles. D'autres lieux s'avèrent en fait de centres de services tels Natashquan.

Parmi ces petits lieux et milieux, certains sont devenus une petite ville telle que Matagami, Lebel-sur-Quévillon, Lac Bouchette, Kuujuaq, Milot, Sacré-Cœur, Labrador City, HavreSaint-Pierre, Malartic, pour répondre aux besoins de main-d'œuvre, de services, de fournitures, de qualité de vie, demandés par l'activité économique principale reliée à l'extraction de la ressource. Lorsqu'ils ont la chance de bénéficier d'une position relativement centrale sur leur territoire de localisation, quelques-uns de ces pôles quaternaires directement ancrés sur le bassin ou le gisement se voient par bonheur alimentés par d'autres activités à rayonnement plus large, comme des commerces plus imposants, des services spécialisés, des administrations gouvernementales.

était constitué par la métropole Montréal autour de laquelle gravite une immense périphérie dont la Gaspésie largement érodée de ses facteurs est devenue l'archétype. Ce 
modèle explicatif ${ }^{7}$ est devenu plus ou moins caduque au fil du temps, notamment lors de la désignation à la fin des années 1960 des pôles régionaux tels que Rimouski, Saguenay, Trois-Rivières, Baie-Comeau, Sherbrooke, Drummondville, Rouyn-Noranda, Gatineau, Rivière-du-Loup, dont chacun possède sa propre périphérie ${ }^{8}$. Ensemble de pôles distants et peu hiérarchisés, qui n'a pas permis d'asseoir convenablement le modèle du « système urbain ». Pourtant utiles, en principe ces deux modèles théoriques se retrouvent ainsi sans beaucoup d'usage fonctionnel pour le Québec. D'autant plus que sous l'angle de la complémentarité, de la cohérence et de la cohésion, la politique gouvernementale préconise pertinemment le modèle du «mariage urbain - rural» dont l'application bute cependant face à l'hégémonie urbaine dans le processus de métropolisation. Bref, le Québec semble en panne d'un schéma global pour modéliser l'intégration de ses composantes territoriales.

\section{Le Québec semble en panne d'un schéma global pour modéliser l'intégration de ses composantes territoriales}

À cet effet, notre lecture de cette dynamique spatio-économique contemporaine du Québec en regard des forces et des tendances qui soutiennent l'occupation des territoires illustre a priori deux schémas bien distincts.
D'une part, se dessine progressivement une «métapole centrale» à multiples formes internes, sous l'impulsion de la tendance lourde à la concentration diffuse ou déconcentrée des activités économiques et de la population. Les métropoles Montréal, Québec et Gatineau de même que les régions métropolitaines de Sherbrooke, Trois-Rivières ainsi que Drummondville, Rivières-du-Loup et Saint-Georges représentent les principaux points d'appui de cette armature métapolitaine qui renferme la grande majorité des activités manufacturières du Québec.

La périphérie nordique offre d'autre part des formes territoriales dessinées par la logique d'accessibilité aux bassins de ressources naturelles ainsi qu'aux collectivités à désenclaver. Ce sont les avant-postes et les corridors qui jouent un rôle important dans l'occupation de ces territoires périphériques à partir d'un arc nordique composé de Sept-Îles, Baie-Comeau, Saguenay, et les pôles de l'Abitibi.

$\mathrm{Au}$ Québec, cette dynamique spatioéconomique duale qui répond aux deux logiques classiques bien connues en théorie, soit la centralité et l'accessibilité, aurait intérêt à bénéficier d'attention, notamment par la mesure des flux de transport des personnes et des marchandises y compris l'hydroélectricité. Questions et hypothèses se posent à cet égard.

\section{Notes et références}

1 Voir Proulx, M.-U. (2006) «La mouvance contemporaine des territoires : la logique spatiale de l'économie au Québec », dans revue Recherches Sociographiques, XLVII, no 3, pp 475-502

2 Voir Scott, A.-J. (editor) (2001) « Global City-Region », Oxford University Press, Oxford, U.K.

3 Voir Alvergne, C. et Latouche, D. (2009) « La métropolisation et la richesse des villes : l'énigme métropolitaine montréalaise », dans Sénécal, G. et Bherer, L. (édit) La métropolisation et ses territoires, Presses de l'Université du Québec. 
4 Voir Proulx, M.U. (2007) « Perspectives géo-économiques de la complémentarité urbaine-rurale au Québec », dans revue Télescope, vol 13, no 3, pp.99-112

5 Voir Ascher, F. (1995) « Métapolis, ou l'avenir de la ville», Éditions Odile Jacob, Paris ; Ascher, F. (2001) « Les nouveaux principes de l'urbanisme », Éditions l'aube, La Tour d'Aigues.

6 Voir Proulx, M.U. (2003) «Polarisation dans la géo-économie du Québec», dans revue Trames, no spécial Villes moyennes et mondialisation sous la direction de Charbonneau, F., Lewis, P. et Manzagol, C. ; et aussi Proulx, M.-U. (2006) «La mouvance contemporaine des territoires: la logique spatiale de l'économie au Québec », dans revue Recherches Sociographiques, XLVII, no 3, pp 475-502

7 Voir le Rapport dit HMR : Higgins, B., Martin, F. et Raynalud, A. (1970) « Les orientations du développement économique régional au Québec », Étude pour le Ministère de l'expansion économique régionale (MEER), Ottawa.

8 Voir Proulx, M.U. (2002) «L’économie des territoires au Québec », Presses de l’Université du Québec, 364 pages. 